\title{
Emotional Intelligence and Day-To-Day Emotion Regulation Processes: Examining Motives for Social Sharing
}

\author{
Micaela Bucich ${ }^{\mathrm{a}}$ \\ Affiliation: School of Psychology, University of Sydney ${ }^{\mathrm{a}}$ \\ Mail: School of Psychology, University of Sydney, Sydney NSW 2007, Australia \\ Email: mbuc6201@uni.sydney.edu.au \\ Carolyn MacCann ${ }^{\text {a }}$ (corresponding author) \\ Affiliation: School of Psychology, University of Sydney ${ }^{a}$ \\ Mail: School of Psychology, University of Sydney, Sydney NSW 2007, Australia \\ Phone: +61 29351 4236, Fax: +61299319199 \\ Email: carolyn.maccann@sydney.edu.au
}

Declarations of interest: none

Funding statement: This research was supported by an Australian Research Council Discovery Grant awarded to the last author (DP150101158).

This manuscript has been accepted for publication in Personality and Individual Differences (https://doi.org/10.1016/j.paid.2018.08.002). This is not the copy of record.

\section{CC-BY-NC-ND}




\title{
Highlights
}

- Self-rated EI significantly predicts 3 emotion regulation processes in daily life

- These processes are social sharing, direct situation modification and reappraisal

- Ability EI does not significantly predict emotion regulation in daily life

- Both self-rated and ability EI predict motives for social sharing in daily life

\begin{abstract}
There is growing interest in the emotion regulation processes that underlie the adaptive functioning of emotionally intelligent individuals. This study uses experience sampling to examine whether the emotional intelligence $(\mathrm{EI})$ of undergraduate students $(N=84)$ relates to their day-to-day use of five emotion regulation processes over a five-day period. We also test whether EI predicts motives for one of the emotion regulation processes (social sharing). We measure both ability EI (the brief Situational Test of Emotion Management) and self-rated EI (the Self-Rated Emotional Intelligence Scale). Self-rated EI significantly predicts more social sharing, direct situation modification and reappraisal. Ability EI does not significantly predict any of the five regulation processes. Both ability and self-rated EI are significantly related to greater bonding and relief motives for social sharing. Self-rated EI is also related to recovery motives. These results suggest that it is the self-beliefs about one's emotional abilities, rather than emotion knowledge, which influence the emotion regulation processes people use in daily life.
\end{abstract}

Key words: emotional intelligence; emotion regulation; social sharing; experience sampling 


\section{Introduction}

Emotional intelligence (EI) and emotion regulation are two conceptually related approaches to understanding the emotional experiences people have. EI describes individual differences in the abilities and traits involved in perceiving, using, understanding, and managing emotions (Mayer, Caruso, \& Salovey, 2016) whereas emotion regulation describes the processes by which people control which emotions they have and when they have them (Gross, 1999). We know that some emotion regulation processes are more effective than others in controlling negative emotions (e.g., perspective taking is generally effective whereas ruminating is generally ineffective; Bushman, 2002; Aldao \& Nolen-Hoeksema, 2010) and that emotionally intelligent people experience fewer negative emotions (SanchezAlvarez, Extremera, \& Fernandez-Berrocal, 2016). What is still largely unclear is whether individual differences in EI relate to differences in the emotion regulation processes people

use. The current research proposes to address this by examining whether ability and self-rated EI predict the use of five different emotion regulation processes in daily life. We also examine whether EI predicts differences in people's motivations for socially sharing their emotions. Given the importance of both EI and emotion regulation to mental health and wellbeing outcomes, our research is relevant for understanding the mechanisms by which person-attributes (EI) translate into behaviours (regulation) known to increase such outcomes.

\subsection{Emotional Intelligence}

While some researchers define EI as a broad set of trait-like variables related to emotion, motivation and social functioning, the current paper uses the commonly accepted Four-Branch Ability Model of EI (Mayer et al., 2016). These four ability branches are: (1) accurate perception of emotion in oneself and others (perception); (2) use of emotions to facilitate problem-solving or task completion (facilitation); (3) understanding how emotions combine and change over time (understanding); and (4) successful regulation of one's own 
and others' emotions (management). This model forms the theoretical basis for both: (a) ability EI, where test-takers must process emotion-related information to answer a question (e.g., judge which of several responses would be most effective in regulating the emotion in a specific situation); and (b) self-rated EI, where test-takers rate how well they think they perceive, use, understand, or manage emotions (e.g., "I know how to keep calm in difficult or stressful situations” (Brackett, Rivers, Shiffman, Lerner, \& Salovey, 2006). In the current study, we examine both self-rated EI and ability EI as predictors of the regulation processes people use in daily life. Our ability EI task measures emotion management, as this skill set is the most complex (requiring skills from the other branches) and most conceptually relevant to emotion regulation (Mayer et al., 2016).

\subsection{Emotion Regulation}

Gross (1999) identifies five families of emotion regulation processes which occur at different points in the emotion-generation process: situation selection, situation modification, attentional deployment, cognitive change, and response modulation. The current study examines five specific regulation processes drawn from four of these families: direct situation modification (taking practical actions to make a direct impact on an emotioneliciting situation), distraction (directing attention away from the emotion-eliciting features of a situation), rumination (directing attention to negative thoughts and feelings, and the causes of these), reappraisal (changing one's thinking about the meaning or self-relevance of an emotion-eliciting situation), and social sharing (recounting an emotion or emotional episode to another person in socially shared language). Direct situation modification is drawn from situation modification, distraction and rumination from emotional deployment, reappraisal from cognitive change, and social sharing from response modulation. In the current study, we use experience sampling to measure emotion regulation (where people 
report their experiences in the moment), thus avoiding the memory biases that can occur in questionnaire research (Csikszentmihalyi \& Larson, 1987).

\subsection{EI and Emotion Regulation}

Many of the abilities required to engage in specific emotion regulation processes are named by Mayer et al. (2016) as critical elements of EI. For example, all types of attention deployment processes logically involve the ability to "prioritize thinking by directing attention" (Mayer et al., p. 294), which is an element of emotion facilitation ability. Both distraction and positive reappraisal require that a person identify the emotion-eliciting element of the situation (so as to divert attention away from it, or re-appraise it), which would require emotion understanding ability. Moreover, using effective regulation processes requires that one plan, monitor and evaluate the processes one is using (so as to use the effective ones more, and ineffective ones less), which are core elements of emotion management ability (Mayer et al., 2016). However, the driver of emotion regulation behaviours may not necessarily be the ability to regulate (ability EI) but also the belief that one has the ability—one's confidence or self-efficacy to use the emotion regulation strategy (self-rated EI). That is, people do things not just because they can, but because they think they can.

There is surprisingly little research on ability EI and regulation. In Peña-Sarrionandia, Mikolajczak, and Gross' (2015) recent summary of the literature linking EI to different emotion regulation strategies: (1) few studies use ability EI (80\% of the findings are based on self-reported EI; most summaries of ability EI rely on the findings of a single study); (2) no studies examine the relationship between EI and social sharing; and (3) no studies examine the EI/emotion regulation relationship using experience sampling to measure regulation in daily life. It is for these reasons that we designed our study to examine both ability and selfrated EI, to use experience sampling, and to focus on social sharing and the motives for it. 
Existing research suggests that EI should relate to higher situation modification, reappraisal and distraction but lower rumination. Specifically, Peña-Sarrionandia et al. (2015) reported that both self-rated and ability EI related to less rumination. Self-rated EI also related to greater direct situation modification, positive reappraisal and greater distraction (there was no research reported for ability EI and these regulation strategies). The largest effect was for reappraisal and the smallest for distraction. Other research has shown that ability EI relates to greater task-focused coping, which is conceptually equivalent to direct situation modification (Davis \& Humphrey, 2012; MacCann, Fogarty, Zeidner, \& Roberts, 2011; Zeidner \& Hadar, 2014). Ability EI also shows a small positive relationship with positive reappraisal (Gohm, Corser, \& Dalsky, 2005; Mestre, Núñez-Lozano, GómezMolinero, Zayas, \& Guil, 2017). While there is no known research on ability EI and distraction, Peña-Sarrionandia et al. (2015) predict positive relationships based on the regulatory advantages of this strategy.

There is also no research on social sharing for either self-rated or ability EI, yet PeñaSarrionandia et al. (2015) posit a negative relationship. They argue that social sharing occurs late in the emotion regulation process (during response modulation), and that people with high EI should have less need for sharing as they use strategies that influence earlier points in the emotional trajectory. However, people with high EI have greater perceived social support (Lopes, Salovey, \& Straus, 2003). They therefore have more opportunities to socially share, given the greater availability of a social network to share with. Moreover, EI relates to seeking social support for both instrumental and emotional reasons (Gohm, et al., 2005; Goldenberg, Matheson, \& Mantler, 2006). Seeking social support plausibly often involves sharing one's feelings about a situation with others to acquire their support. We therefore hypothesize that higher EI would be linked with greater social sharing, counter to PeñaSarrionandia et al. (2015). 


\subsection{Different Types of Social Sharing}

One reason it is difficult to predict the EI/social sharing relationship is the complex nature of social sharing. Rimé (2009) proposed a two-mode theory of social sharing. The cognitive mode helps the sharer progress towards emotional recovery whereas the socioaffective mode brings only a sense of temporary relief. Recovery is more likely when the sharing partner helps the sharer see things from a new perspective. Relief results from the sharing partner providing validation, comfort and support. Sharing most commonly develops in the socio-affective mode and the cognitive mode occurs much more rarely (Rimé, 2007).

Measuring the mode of social sharing is difficult as it relies on the sharing partner as well as the sharer. However, the sharer's motivations should play a key role in which mode is used. In the current study, we consider three types of motivation: recovery motives (sharing to gain new perspectives or advice); relief motives (sharing to vent or arouse empathy) and bonding motives (sharing to strengthen social bonds) (Duprez et al., 2015). Recovery motives and relief motives conceptually link to the cognitive and socio-affective modes of social sharing respectively. As relief motives are frequently reported (Rime, 2007; Duprez et al., 2015) and feeling understood is a basic need (Kennedy-Moore \& Watson, 1999), we do not believe relief motives will relate to EI. Recovery motives, while used rarely, are more adaptive and might therefore be used more often by emotionally intelligent people. Bonding motives were considered important for inclusion in the current study because high EI people may socially share in order to maintain their intimate relationships (Lopes et al., 2003), rather than for regulatory purposes alone.

\subsection{Study Hypotheses}

Hypothesis 1: EI will be significantly related to emotion regulation processes, showing a positive relationship with direct modification, distraction, reappraisal and social 
sharing, and a negative relationship with rumination. These relationships will hold for both ability EI (Hypothesis 1a) and self-rated EI (Hypothesis 1b).

Hypothesis 2: EI will be significantly positively related to bonding and recovery motives for social sharing but not significantly related to relief motives. These relationships will hold for both ability EI (Hypothesis 2a) and self-rated EI (Hypothesis 2b).

\section{Method}

\subsection{Participants}

Participants were 84 undergraduate psychology students (62 female, Mean age $=19.7$, $\mathrm{SD}=4.9$ ), who volunteered to participate for course credit after reading the study advertisement on the university's participant recruitment system. Reported ethnicities were White (62\%), Asian (31\%) and Other/unspecified (7\%). An additional 37 participants were recruited but not included in the final study (see supplementary material for more information on data screening).

\subsection{Procedure}

Participants first came to a lab session where they registered their smartphone details in the survey distribution system SurveySignal; then completed the test battery below (measures were randomly ordered). The following week, participants received 15 SMS messages from SurveySignal ( 3 per day). Each contained a link to a 2-minute mini-survey (described below) to complete on a smartphone. After completing the experience samples, participants were granted course credit and emailed a debrief statement. Participants completed between 4 and 18 mini-surveys (median $=11 ; 4$ participants completed $>15$ surveys due to answering the same SMS message more than once). 


\subsection{Measures}

\section{Test Battery}

2.3.1. Demographic questionnaire. Participants reported their date of birth, gender, country of birth, ethnicity and English-speaking ability.

\subsubsection{Situational Test of Emotion Management - Brief (STEM-B; Allen et al.,}

2015; 18 items). This maximum-performance test of emotion management used consensus scoring. Example item: Clayton has been overseas for a long time and returns to visit his family. So much has changed that Clayton feels left out. What action would be the most effective for Clayton? (a) Nothing - it will sort itself out soon enough. (b) Tell his family he feels left out. (c) Spend time listening and getting involved again. (d) Reflect that relationships can change with time.

\subsubsection{Self-Rated Emotional Intelligence Scale (SREIS; Brackett et al., 2006; 19}

items). Participants rate how accurately 19 statements describe them from 'very inaccurate' (1) to 'very accurate' (5) (e.g. "I know how to keep calm in difficult or stressful situations").

\section{Experience Sampling Survey}

All items were rated from 1 (Not at all) to 6 (Very much).

2.3.4. Regulatory Processes (10 items). Participants were asked "Since the last survey, how much have you done the following in response to your feelings?". Items represented direct situation modification (“changed something in your environment?", "taken steps to change the situation you were in?"), distraction ("engaged in activities to distract yourself from your feelings?”, “diverted your attention away from your feelings?”), rumination ("ruminated or dwelled on your emotions or what caused them", "been unable to stop thinking about your feelings?"), reappraisal ("looked at things from a different perspective?", “changed the way you were thinking about what caused your feelings?"), and social sharing ("talked to others about your feelings or the situation that caused them?", "texted, emailed 
or used social media to communicate how you feel with others?"). Items were taken from Brans et al. (2013) and Haines et al. (2016), modified slightly for consistency across items.

2.3.5. Social Sharing Motives (10 items). Social sharing motives items were presented after the regulatory processes items, beginning with 'I discussed my feelings to...'. There were four recovery items (e.g., "get an outside perspective"); four relief items (e.g., "let off steam") and two bonding motives (e.g., "stay in touch with others"). Items were taken from Duprez et al. (2015) and Carver, Scheier, and Weintraub (1989). ${ }^{1}$

\section{Results}

Multi-level correlations were performed in Mplus, clustering experience samples within participants (Level $2 N=84$; Level $1 N=956$; average cluster size $=11.4$ ).

3.1. Descriptive statistics and reliabilities. Descriptive statistics and Cronbach's alpha reliability estimates for all level 2 variables are reported in Table 1. Reliability ranged from .66 (STEM-B) to .98 (Level 2 recovery motives).

\footnotetext{
${ }^{1}$ Although not relevant to the current hypotheses, big five personality was also measured, and each mini-survey included four affect items (frustration, happiness, sadness and contentment).
} 
Table 1.

Descriptive statistics, reliability, and Pearson correlations among study variables (correlations in parentheses correct for unreliability)

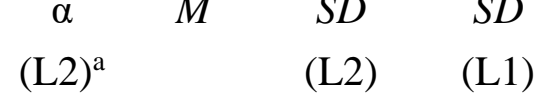

EI

$\begin{array}{llllll}\text { 1. STEM } & .66 & 0.61 & 0.08 & - & \\ \text { 2. SREIS } & .83 & 3.66 & 0.48 & - & .36^{* *}\left(.49^{* *}\right)\end{array}$

Experience Sampling Variables

Emotion Regulation

\begin{tabular}{|c|c|c|c|c|c|c|c|c|c|c|c|c|c|c|c|}
\hline 3. Direct Modification & .87 & 2.63 & .83 & 2.17 & .33 & $.01(.01)$ & $.24^{*}\left(.28^{* *}\right)$ & - & $.40^{* *}$ & $.25^{* *}$ & $.47^{* *}$ & $.34^{* *}$ & $.25^{* *}$ & $.19^{* * *}$ & $.21^{* *}$ \\
\hline 4. Distraction & .92 & 2.90 & .92 & 2.32 & .35 & $.03(.04)$ & $.03(.03)$ & $.64^{* *}$ & & $.29^{* *}$ & $.35^{* *}$ & $.29^{* *}$ & $24^{* *}$ & $.27^{* * *}$ & $.09^{* *}$ \\
\hline 5. Rumination & .79 & 2.43 & .75 & 2.11 & .29 & $.02(.03)$ & $.14(.17)$ & $.54^{* *}$ & $.53^{* *}$ & & $.37^{* *}$ & $.44^{* *}$ & $.33^{* *}$ & $.37^{* * *}$ & .06 \\
\hline 6. Reappraisal & .89 & 2.54 & .83 & 2.03 & .36 & $-.01(-.01)$ & $.25^{*}\left(.29^{* *}\right)$ & $.82^{* *}$ & $.55^{* *}$ & $.66^{* *}$ & - & $.40^{* *}$ & $.50^{* *}$ & $.37^{* * *}$ & $.22^{* *}$ \\
\hline 7. Social Sharing & .80 & 2.27 & .81 & 2.37 & .26 & $.15(.21)$ & $.48^{* *}\left(.59^{* *}\right)$ & $.59^{* *}$ & $.29^{* *}$ & $.54^{* *}$ & $.65^{* *}$ & - & $.49^{* *}$ & $.53^{* *}$ & $.37^{* *}$ \\
\hline \multicolumn{16}{|c|}{ Motives for Social Sharing } \\
\hline 8. Recovery & .98 & 2.66 & 1.14 & 4.25 & .47 & $.07(.09)$ & $.31^{* *}\left(.34^{* * *}\right)$ & $.49^{* *}$ & $.22^{*}$ & $.56^{* *}$ & $.68^{* *}$ & $.82^{* *}-$ & & $.68^{* *}$ & $.36^{* *}$ \\
\hline 9. Relief & .92 & 2.41 & .96 & 3.88 & .42 & $.17\left(.22^{*}\right)$ & $.20\left(.23^{*}\right)$ & $.48^{* *}$ & $.30^{* *}$ & $.62^{* *}$ & $.60^{* *}$ & $.72^{* *}$ & $.79^{* *}$ & & $.30^{* *}$ \\
\hline 10. Bonding & .96 & 2.45 & 1.09 & 2.10 & .48 & $.30^{* *}\left(.38^{* *}\right)$ & $.20\left(.22^{*}\right)$ & $.45^{* *}$ & $.34^{* *}$ & $.39^{* *}$ & $.48^{* *}$ & $.59^{* *}$ & $.51^{* *}$ & $.56^{* *}$ & \\
\hline
\end{tabular}

Note. ICC $=$ intraclass correlation. Within-person correlations above diagonal, between-person correlations below diagonal.

${ }^{a}$ Cronbach's alpha for the Level 2 experience sampling variables was calculated in SPSS using the average value of each item across all experience samples

${ }^{*} p<.05,{ }^{* *} p<.01$ 
3.2. Hypothesis 1 (EI will relate to emotion regulation processes). Correlations of all Level 2 variables are given in Table 1. Because reliability was low for the STEM-B, we calculated the EI/regulation and EI/motives correlations correcting for reliability (Spearman, 1904; corrected results in parentheses). We test hypotheses with respect to the corrected estimates.

The STEM-B was not significantly correlated with any emotion regulation process, such that Hypothesis 1a was not supported. SREIS scores were significantly and positively correlated with direct modification, reappraisal and social sharing but not significantly related to rumination or distraction. The correlation between the SREIS and social sharing was of medium to large effect size, whereas the correlations of the SREIS with both direct modification and reappraisal were small to medium in size. Results provide partial support for Hypothesis $1 b$.

3.3. Hypothesis 2 (EI will predict social sharing motives). SREIS scores were significantly associated with all three motives for social sharing, and STEM-B scores were significantly associated with relief and bonding motives. This result provided mixed support for Hypothesis 2, as we expected significant correlations with bonding and recovery motives, but not relief motives. 


\section{Discussion}

Results show that self-rated EI predicted emotion regulation processes in daily life but ability EI did not, supporting Hypothesis $1 \mathrm{~b}$ but not 1a. The largest effect was found for social sharing, where self-rated EI predicted greater sharing. Both self-rated and ability EI predicted motives for social sharing. Self-rated EI predicted all three motives (recovery, bonding, and relief, with the strongest effect for recovery) whereas ability EI predicted greater bonding and relief motives, but not recovery motives.

\subsection{EI and Emotion Regulation Processes}

The present findings show no evidence of a relationship between ability EI and emotion regulation in daily life. Only self-rated EI was related to emotion regulation. This is consistent with the results of Pena-Sarrionandia et al.'s (2015) review, where emotion regulation was more strongly related to EI rating scales than to ability EI.

While emotion knowledge is important, researchers acknowledge that other factors are needed to motivate emotion regulation (Mayer et al., 2016). Various researchers have stressed that belief in one's emotional abilities can determine whether or not one uses these abilities (Kirk et al. 2008). Our research supports this idea-emotion regulation behaviours were more strongly linked to self-perceptions of emotional abilities than to actual emotional abilities. For emotion regulation, knowing what to do appears less important than believing one can do it. Self-rated EI is sometimes referred to as 'emotional self-efficacy' (Petrides, Perez-Gonzalez, \& Furnham, 2007). Given well-known theory that self-efficacy determines the initiation and persistence with coping behaviour (Bandura, 1977), it is not surprising that emotional selfefficacy determines how much emotion regulation activity is engaged in.

Our results may help to explain the consistent meta-analytic findings that self-rated EI has stronger prediction than ability EI across a range of positive outcomes, including job performance, health, and wellbeing (Joseph \& Newman, 2010; Martins, Ramalho, \& Morin, 
2010; Sanchez-Alvarez et al., 2016). One of the possible mechanisms by which EI translates into positive outcomes is that high EI enables better emotion regulation (e.g., people who can better regulate their emotions at work will perform better as their anxiety or stress does not impede their performance). If self-rated EI (but not ability EI) is a determining factor in the emotion regulation processes people use, this would explain why self-rated EI shows stronger prediction of positive outcomes. However, it is also true that most outcome measures are based on rating-scales (either self-ratings or employer ratings), such that the stronger prediction from self-rated EI than ability EI may represent shared method variance.

The strong association between self-rated EI and social sharing is plausibly a bidirectional relationship. Believing one's emotional abilities are high would provide confidence to use them (such that higher EI causes greater social sharing). However, people may gain confidence in their emotional abilities by sharing their emotions with others more in their daily lives (such that greater social sharing increases self-rated EI).

The positive relationships found between self-rated EI and both situation modification and reappraisal are in line with previous research (Goldenberg et al., 2006). The nonsignificant relationship between EI and rumination is surprising as rumination is known to intensify negative emotions (Bushman, 2002) and is characteristic of a number of mental disorders (Aldao \& Nolen-Hoeksema, 2012). Distraction was expected to share a positive relationship with EI as it can be an easy and effective way of managing negative emotions so that one can stay on task (Brans et al., 2013). However, it may be that because distraction is an 'easy' way of dealing with emotions, higher levels of EI are not required to use this (Brans et al., 2013).

\subsection{Emotional Intelligence and Motives for Social Sharing}

Results provided mixed support for Hypothesis 2 (the EI/social sharing motives relationship). While self-rated EI was significantly associated with both recovery and bonding 
motives (as hypothesized) and ability EI was significantly associated with bonding motives (as hypothesized), both self-rated and ability EI were also significantly related to relief motives (which was not hypothesized) and ability EI was not related to recovery motives. All relationships with motives were small to moderate.

The recovery motive items used in the current study relate to gaining advice or a new perspective on an emotion-eliciting situation. The fact that ability EI was not related to recovery motives, may indicate that high ability EI individuals already have knowledge of what to do, such that they do not have any greater motivation to gain advice. On the other hand, people with high self-rated EI were motivated by recovery motives, as well as by relief and bonding motives.

Ability EI was most strongly associated with bonding motives for social sharing. Willingness to share emotions is related to a greater number of more intimate relationships (Graham, Huang, Clark, \& Helgeson, 2008) and the present findings suggest that people with higher ability EI are more aware of these social benefits. Social sharing could partially explain why those higher in EI have been found to report more satisfying relationships than those lower in EI (Lopes et al., 2003). Future research could test social sharing as a mediator of these outcomes.

The unexpected positive relationships between EI and relief motives may be explained by high EI people being more aware of their basic need to feel understood (Kennedy-Moore \& Watson, 1999). That is, rather than people with higher EI actually having greater relief motives for social sharing, they may just be more aware of these underlying motives than those lower in EI.

\subsection{Limitations and Future Directions}

There were some limitations with the current data set that precluded more complex analyses. We had a relatively small sample size, and a possible ceiling effect on the STEM-B 
which may have compromised reliability (although we attempted to account for this by correcting for attenuated reliability). The small within-person $N$ (a median of 11 data points per person) also meant that there was not enough power to look at cross-level moderation with confidence (e.g., whether EI predicted the relationship between social sharing motivations and the amount of social sharing engaged in).

This study relied on self-ratings of emotion regulation processes and motives for social sharing, as well as the self-report measure of EI. As such, stronger correlations with emotion regulation for self-rated EI (as compared to ability EI) may represent a method effect. However, our ability EI task measured emotion management only whereas our selfreport EI scale measured all four branches. It is possible that other branches may relate to emotion regulation processes. For example, understanding the causes of negative emotions (the understanding branch) may make people less likely to engage in processes that may cause or prolong negative affect, such a rumination or venting. As such, it is possible that a difference in construct breadth (rather than method-of-measurement) may explain differences between self-report and ability EI in this study. Follow-up studies should be conducted with a more comprehensive ability-based assessment of EI.

Using self-reports in the experience sampling survey may have introduced response biases such as social desirability, cognitive biases, and cultural norms in reporting emotion regulation and motives for social sharing. (Scollon, Kim-Prieto, \& Diener, 2003). In addition, it meant that only conscious emotion regulation processes were captured. There is a growing interest in unconscious emotion regulation (Hopp, Troy, \& Mauss, 2011) and in the more unconscious processes underlying EI (Fiori, 2009). A challenge for future research will be to explore the relationship between EI and automatic emotion regulatory processes. 


\subsection{Conclusion}

The current study examined the relationship between EI and the amount that five emotion regulation processes are used in daily life. While both self-rated and ability EI were important in determining motives for social sharing, the degree to which emotion regulation processes were used seemed clearly linked to having belief in one's emotional abilities rather than having knowledge of effective emotion regulation processes. 


\section{References}

Aldao, A., and Nolen-Hoeksema, S. (2012). When are adaptive strategies most predictive of psychopathology? Journal of Abnormal Psychology,121, 276-281. doi: $10.1037 / \mathrm{a} 0023598$

Allen, V., Rahman, N., Weissman, A., MacCann, C., Lewis, C., \& Roberts, R.D. (2015). The situational test of emotional management - brief (STEM-B): Development and validation using item response theory and latent class analysis. Personality and Individual Differences, 81, 195-200. doi:10.1016/j.paid.2015.01.053

Bandura, A. (1977). Self-efficacy: toward a unifying theory of behavioral change. Psychological Review, 84, 191-215. doi:10.1037/0033-295X.84.2.191

Brackett, M.A., Rivers, S. E., Shiffman, S., Lerner, N., \& Salovey, P. (2006). Relating emotional abilities to social functioning: A comparison of self-report and performance measures of emotional intelligence. Journal of Personality and Social Psychology, 91, 780-795. doi: 10.1037/0022-3514.91.4.780

Brans, K., Koval, P., Verduyn, P., Lim, Y.L., \& Kuppens, P. (2013). The regulation of negative and positive affect in daily life. Emotion, 13, 926-939. doi:10.1037/a0032400

Bushman, B.J. (2002). Does venting anger feed or extinguish the flame? Catharsis, rumination, distraction, anger, and aggressive responding. Personality and Social Psychology Bulletin, 28, 724-731. doi: 10.1177/0146167202289002

Carver, C.S., Scheier, M.F., \& Kumari Weintraub, J. (1989). Assessing coping strategies: A theoretically based approach. Journal of Personality and Social Psychology, 56, 267-283. doi:10.1037/0022-3514.56.2.267 
Csikszentmihalyi, M., \& Larson, R. (1987). Validity and reliability of the experiencesampling method. The Journal of Nervous and Mental Disease, 175, 526-536. doi:10.1097/00005053-198709000-00004

Davis, S.K., \& Humphrey, N. (2012). The influence of emotional intelligence (EI) on coping and mental health in adolescence: Divergent roles for trait and ability EI. Journal of Adolescence, 35, 1369-1379. doi: 10.1016/j.adolescence.2012.05.007

Duprez, C., Christophe, V., Rimé, B., Congard, A., \& Antoine, P. (2015). Motives for the social sharing of an emotional experience. Journal of Social and Personal Relationships, 32, 757-787. doi:10.1177/0265407514548393

Fiori, M. (2009). A new look at emotional intelligence: A dual-process framework. Personality and Social Psychology Review, 13, 21-44. doi:10.1177/1088868308326909

Gohm, C.L., Corser, G.C., \& Dalsky, D.J. (2005). Emotional intelligence under stress: Useful, unnecessary, or irrelevant? Personality and Individual Differences, 39(6), 1017-1028. doi: 10.1016/j.paid.2005.03.018

Goldenberg, I., Matheson, K., \& Mantler, J. (2006). The assessment of emotional intelligence: A comparison of performance-based and self-report methodologies. Journal of Personality Assessment, 86, 33-45. doi: 10.1207/s15327752jpa8601_05

Graham, S.M., Huang, J.Y., Clark, M.S., \& Helgeson, V.S. (2008). The positives of negative emotions: Willingness to express negative emotions promotes relationships. Personality and Social Psychology Bulletin, 34, 394-406. doi:10.1177/0146167207311281

Gross, J.J. (1999). Emotion regulation: Past, present, future. Cognition \& Emotion, 13, 551573. doi: 10.1080/026999399379186 
Hopp, H., Troy, A.S., \& Mauss, I.B. (2011). The unconscious pursuit of emotion regulation: Implications for psychological health. Cognition \& Emotion, 25, 532-545. doi:10.1080/02699931.2010.532606

Joseph, D.L., \& Newman, D.A. (2010). Emotional Intelligence: An Integrative Meta-Analysis and Cascading Model. Journal of Applied Psychology, 95, 54-78. doi: $10.1037 / \mathrm{a} 0017286$

Kennedy-Moore, E., \& Watson, J.C. (1999). Expressing emotion: Myths, realities, and therapeutic strategies. New York: London, Guilford Press.

Kirk, B. A., Schutte, N.S., \& Hine, D.W. (2008). Development and preliminary validation of an emotional self-efficacy scale. Personality and Individual Differences, 45, 432436. doi:10.1016/j.paid.2008.06.010

Lopes, P.N., Salovey, P., \& Straus, R. (2003). Emotional intelligence, personality, and the perceived quality of social relationships. Personality and Individual Differences, 35, 641-658. doi:10.1016/S0191-8869(02)00242-8

MacCann, C., Fogarty, G.J., Zeidner, M., \& Roberts, R.D. (2011). Coping mediates the relationship between emotional intelligence (EI) and academic achievement. Contemporary Educational Psychology, 36, 60-70. doi:10.1016/j.cedpsych.2010.11.002

Martins, A., Ramalho, N., \& Morin, E. (2010). A comprehensive meta-analysis of the relationship between Emotional Intelligence and health. Personality and Individual Differences, 49, 554-564. doi: 10.1016/j.paid.2010.05.029

Mayer, J.D., Caruso, D.R., \& Salovey, P. (2016). The ability model of emotional intelligence: Principles and updates. Emotion Review, 8, 290-300. doi: 10.1177/1754073916639667 
Mestre, J.M., Núñez-Lozano, J.M., Gómez-Molinero, R., Zayas, A., \& Guil, R. (2017).

Emotion regulation ability and resilience in a sample of adolescents from a suburban area. Frontiers in psychology, 8. doi:10.3389/fpsyg.2017.01980

Peña-Sarrionandia, A., Mikolajczak, M., \& Gross, J. J. (2015). Integrating emotion regulation and emotional intelligence traditions: A meta-analysis. Frontiers in Psychology, 6, 160. doi:10.3389/fpsyg.2015.00160

Petrides, K., Perez-Gonzalez, J. C., \& Furnham, A. (2007). On the criterion and incremental validity of trait emotional intelligence. Cognition and Emotion, 21(1), 26-55. doi: $10.1080 / 02699930601038912$

Rimé, B. (2007). "Interpersonal emotion regulation," in J. J. Gross (Ed.) Handbook of Emotion Regulation (pp. 466-485), New York, NY: Guilford Press.

Rimé, B. (2009). Emotion elicits the social sharing of emotion: Theory and empirical review. Emotion Review, 1, 60-85. doi:10.1177/1754073908097189

Sanchez-Alvarez, N., Extremera, N., \& Fernandez-Berrocal, P. (2016). The relation between emotional intelligence and subjective well-being: A meta-analytic investigation. The Journal of Positive Psychology, 11, 276-285. doi:10.1080/17439760.2015.1058968

Scollon, C.N., Kim-Prieto, C., \& Diener, E. (2003). Experience sampling: Promises and pitfalls, strengths and weaknesses. Journal of Happiness Studies, 4, 5-34. doi:10.1023/A:1023605205115

Spearman, C. (1904). The proof and measurement of association between two things. The American Journal of Psychology, 15, 72-101.

Zeidner, M., \& Hadar, D. (2014). Some individual difference predictors of professional wellbeing and satisfaction of health professionals. Personality and Individual Differences, 65, 91-95. doi: 10.1016/j.paid.2014.01.032 


\section{SUPPLEMENTARY MATERIAL}

Experience Sampling Items

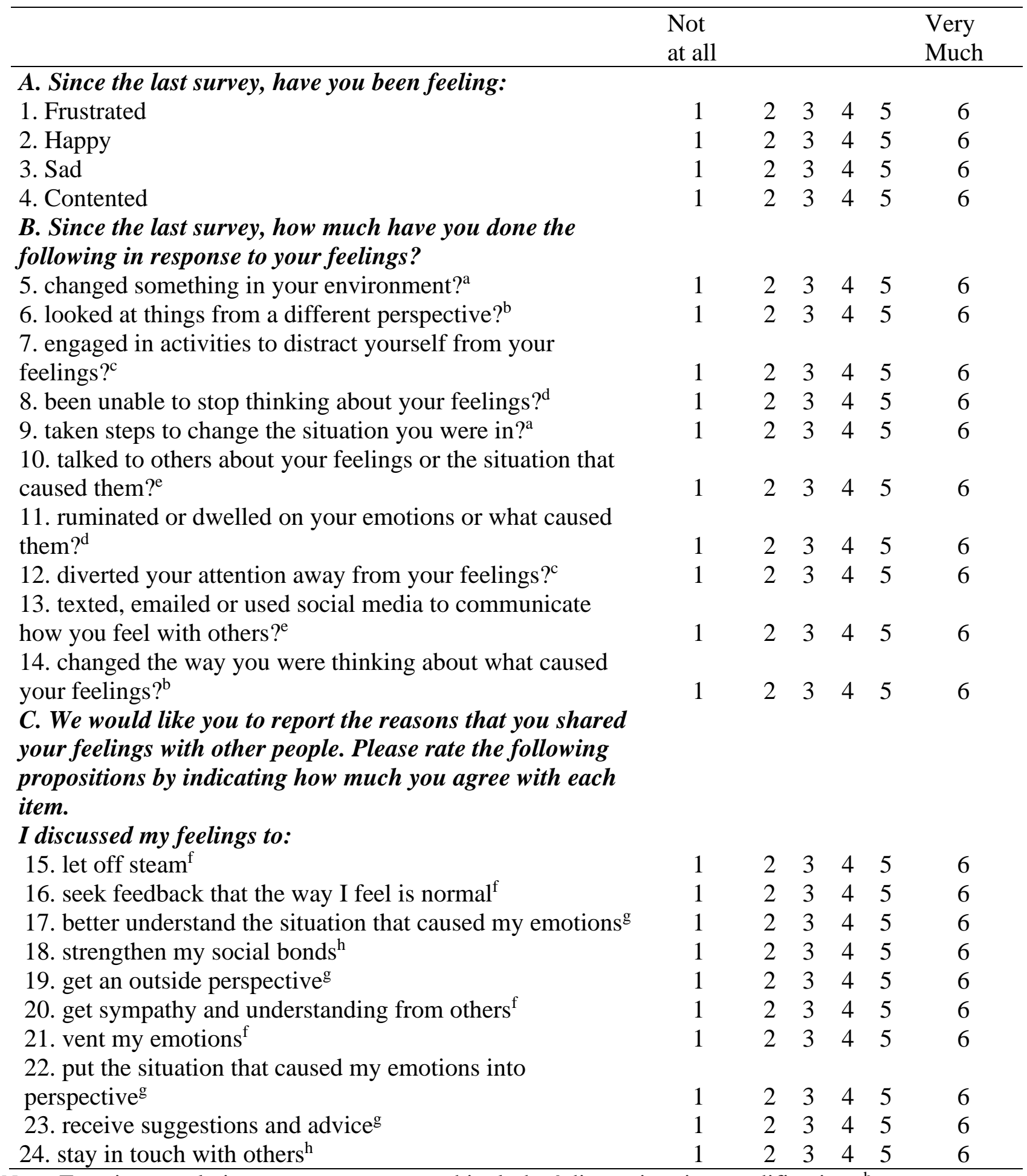

Note. Emotion regulation processes measured include: ${ }^{a}$ direct situation modification, ${ }^{\mathrm{b}}$

reappraisal, ${ }^{\mathrm{c}}$ distraction, ${ }^{\mathrm{d}}$ rumination, and ${ }^{\mathrm{e}}$ social sharing. Motives for social sharing include:

${ }^{\mathrm{f}}$ relief motives, ${ }^{\mathrm{g}}$ recovery motives, and ${ }^{\mathrm{h}}$ bonding motives. 